\title{
diagnósticos de ENFERMAgem de PACIENTES NO PERÍOdO TRANSOPERATÓRIO DE CIRURGIA CARDÍACA ${ }^{1}$
}

\author{
Luzia Elaine Galdeano ${ }^{2}$ \\ Lídia Aparecida Rossi ${ }^{3}$ \\ Luciane Facio Nobre ${ }^{4}$ \\ Daniela Sarreta Ignácio ${ }^{4}$
}

Galdeano LE, Rossi LA, Nobre LF, Ignácio DS. Diagnósticos de enfermagem de pacientes no período transoperatório de cirurgia cardíaca. Rev Latino-am Enfermagem 2003 março-abril; 11(2):199-206.

O objetivo deste estudo foi identificar os diagnósticos de enfermagem de pacientes no período transoperatório de cirurgia cardíaca. Para a coleta de dados, foi elaborado e validado um instrumento baseado no modelo conceitual de Horta. Foram avaliados 17 pacientes adultos, no período transoperatório de cirurgia cardíaca, sendo nove homens e oito mulheres, com idade média de 58 anos. Os diagnósticos de enfermagem, identificados segundo a Taxonomia I da NANDA, com freqüência superior a 50,0\%, foram: risco para infecção (100\%), risco para desequilíbrio de volume de líquidos (100\%), troca de gases prejudicada (100\%), risco para aspiração (100\%), proteção alterada (100\%), integridade da pele prejudicada (100\%), risco para disfunção neurovascular periférica (100\%), risco para lesão perioperatória de posicionamento (100\%) e risco para temperatura corporal alterada (94,1\%). Observamos uma predominância dos diagnósticos de enfermagem relacionados às necessidades fisiológicas.

DESCRITORES: diagnóstico de enfermagem, cirurgia torácica, assistência perioperatória

\section{NURSING DIAGNOSIS IN THE INTRA-OPERATIVE PERIOD OF CARDIAC SURGERY}

The purpose of this study was to identify nursing diagnoses of patients in the intra-operative period of cardiac surgery. One instrument was elaborated and validated for data collection. Such instrument was organized according to The Theory of Basic Human Needs. Seventeen adult patients in the intra-operative period of cardiac surgery were evaluated, of whom 09 were males and 08 were females with the average of 58 years old. Following data collection, the nursing diagnoses were identified based on NANDA's Taxonomy I. The nursing diagnoses identified with a higher frequency than 50\% were: Risk for infection (100\%), Risk for fluid volume imbalance (100\%), Impaired gas exchange (100\%), Risk for aspiration (100\%), Altered protection (100\%), Impaired skin integrity (100\%), Risk for peripheral neurovascular dysfunction (100\%), Risk for perioperative positioning injury (100\%) and Risk for altered body temperature (94.1\%). It was identified a predominance of the diagnoses related to psychobiological needs.

DESCRIPTORS: nursing diagnosis, thoracic surgery, perioperative care

\section{DIAGNÓSTICO DE ENFERMERÍA EN EL PERIOdO TRANS-OPERATORIO DE CIRUGIA CARDIACA}

El objetivo de este estudio fué identificar los diagnósticos de enfermería en pacientes de cirugía cardiaca en el periodo trans operatorio. Para la colecta de datos fué elaborado y validado un instrumento baseado en el modelo conceptual de Horta. Fueron evaluados 17 pacientes adultos de cirugía cardiaca en el periodo preoperatorio, siendo nueve hombres y ocho mujeres, con edad media de 58 años. Los diagnósticos de enfermería, identificados segundo Taxonomia I de la NANDA con frecuencia superior a 50,0\% fueron: Riesgo para infección (100\%), Riesgo para desequilibrio de volume de líquidos (100\%), Cambio de gases perjudicado (100\%), Riesgo para aspiración (100\%), Protección alterada (100\%), Integridad de piel perjudicada (100\%), Riesgo para disfunción neurovascular periférica (100\%), Riesgo para lesión perioperatoria de posicionamiento (100\%) y Riesgo para temperatura corporal alterada (94,1\%). Observamos uma predominancia de diagnósticos de enfermería relacionados a las necesidades psicobiológicas.

DESCRIPTORES: diagnostico de enfermeria, cirurgia toracica, atencion perioperativa

\footnotetext{
${ }^{1}$ Este estudo foi realizado com o apoio da Fundação de Amparo à Pesquisa do Estado de São Paulo - FAPESP e constitui parte de uma pesquisa intitulada Diagnósticos de enfermagem de pacientes no período perioperatório de cirurgia cardíaca apresentada ao Programa de PósGraduação, Área Fundamental, da Escola de Enfermagem de Ribeirão Preto da Universidade de São Paulo; ${ }^{2}$ Mestre em Enfermagem, Professor Assistente da Faculdade de Enfermagem do Hospital Israelita Albert Einstein, e-mail: legaldeano@hotmail.com; ${ }^{3}$ Professor Associado; ${ }^{4}$ Aluna do Curso de Especialização em Cardiologia da Disciplina de Enfermagem Clínico-Cirúrgica. Escola de Enfermagem de Ribeirão Preto da Universidade de São Paulo, Centro Colaborador da OMS para o desenvolvimento da pesquisa em enfermagem
} 
INTRODUÇÃO

As doenças cardiovasculares têm se apresentado, nas últimas décadas, em proporções expressivas dentre as causas de morbidade e mortalidade, tanto nos países desenvolvidos como naqueles em desenvolvimento. No Brasil, é a principal causa de morte, vitimando 300.000 brasileiros por ano ${ }^{(1)}$.

A cirurgia cardíaca é realizada quando a probabilidade de uma vida útil é maior com o tratamento cirúrgico do que com o tratamento clínico. Existem três tipos de cirurgia cardíaca: as corretoras (fechamento de canal arterial, de defeito de septo atrial e ventricular), as reconstrutoras (revascularização do miocárdio, plastia de valva aórtica, mitral ou tricúspide) e as substitutivas (trocas valvares e transplantes).

O tipo mais comum de cirurgia cardíaca reconstrutora é a revascularização do miocárdio. Nela, um vaso sangüíneo (geralmente a veia safena e/ou a artéria mamária interna) é anastomosado com a artéria coronária, distal ao ponto ocluído, e a aorta ascendente, de forma a isolar o local do vaso obstruído e restabelecer a perfusão da artéria coronária. O objetivo da revascularização do miocárdio é aliviar a angina e preservar a função do miocárdio.

O período transoperatório, que corresponde ao momento em que o paciente é recebido no Centro Cirúrgico até o momento de sua transferência para a Unidade de Recuperação Anestésica, é normalmente considerado um período crítico para o paciente, especialmente em cirurgia cardíaca, devido à complexidade da cirurgia e procedimentos a ela inerentes, como, por exemplo, a circulação extracorpórea (CEC), e ao prolongado tempo intra-operatório. É um período caracterizado por mudanças fisiológicas geradas pelas condições impostas pela cirurgia cardíaca, CEC e anestesia, podendo levar a complicações no pós-operatório.

A CEC é um procedimento realizado na maioria das cirurgias cardíacas, em que a máquina realiza um desvio cardiopulmonar total, isto é, desvia o sangue das veias cavas para um reservatório e reinfunde-o após a oxigenação artificial de volta à aorta, substituindo, temporariamente, as funções de bombeamento do coração e ventilatórias dos pulmões.

A experiência profissional das pesquisadoras, enquanto enfermeiras assistenciais de pacientes com problemas cardíacos, gerou inquietações relacionadas ao tipo de assistência de enfermagem prestada a esses pacientes. A complexidade de cuidados requeridos por pacientes que se encontram no período perioperatório de cirurgia cardíaca, cujas condições de saúde podem variar de minuto a minuto, necessitando de intervenções de enfermagem fundamentadas em um método que privilegia a tomada de decisão, despertou o interesse em organizar a assistência de enfermagem com base no processo de enfermagem.

No âmbito estadual, a assistência de enfermagem parece estar passando por um período de mudanças, uma vez que já existe a preocupação dos profissionais em embasar a assistência prestada no processo de enfermagem. Essas mudanças foram fortalecidas pela decisão do Conselho Regional de Enfermagem do Estado de São Paulo em normatizar a implementação da Sistematização da Assistência de Enfermagem - SAE nas instituições de Saúde e, com isso, fazer com que toda a classe profissional se conscientize da importância em redefinir sua prática, baseando suas ações em valores éticos e organizando-as de forma a possibilitar a continuidade da assistência ${ }^{(2)}$.

O processo de enfermagem é constituído de um conjunto de etapas: coleta de dados, diagnóstico de enfermagem, planejamento, implementação e avaliação, que focalizam a individualização do cuidado mediante uma abordagem de solução de problemas a qual se fundamenta em teorias e modelos conceituais de enfermagem ${ }^{(3)}$. Dentre essas etapas, o diagnóstico de enfermagem tem merecido destaque por se tratar de uma etapa dinâmica, sistemática, organizada e complexa do processo de enfermagem, significando não apenas uma simples listagem de problemas, mas uma fase que envolve avaliação crítica e tomada de decisão ${ }^{(4)}$.

Na literatura nacional, encontramos estudos que se reportam à identificação dos diagnósticos de enfermagem nos períodos pré e pós-operatórios de cirurgia cardíaca, no entanto, o mesmo não ocorreu para o período transoperatório de cirurgia cardíaca ${ }^{(5-7)}$. Um estudo analisou, mediante uma extensa revisão de literatura, a incidência dos diagnósticos de enfermagem no período perioperatório. Os diagnósticos de enfermagem identificados nesse estudo estão relacionados a seguir, em ordem decrescente. São eles: "risco para lesão perioperatório de posicionamento, risco para infecção, proteção alterada, dor, risco para disfunção neurovascular periférica, risco para aspiração, risco para integridade da pele prejudicada, risco para temperatura corporal alterada, risco para injúria, ansiedade, medo, hipotermia, integridade da pele prejudicada, mobilidade física prejudicada, risco para déficit de volume de líquidos e déficit de conhecimento"(8).

O presente estudo teve por objetivo identificar os diagnósticos de enfermagem no período transoperatório de cirurgia cardíaca, com o intuito subsidiar o planejamento do cuidado nesse período, considerando as necessidades individuais do paciente.

\section{METODOLOGIA}

Este estudo constitui parte de uma pesquisa intitulada Diagnósticos de enfermagem de pacientes no 
período perioperatório de cirurgia cardíaca, aprovada pelo Comitê de Ética e Pesquisa do Hospital das Clínicas da Faculdade de Medicina de Ribeirão Preto-USP.

O estudo foi realizado na unidade de internação cirúrgica e unidade de internação clínica do Hospital das Clínicas da Faculdade de Medicina de Ribeirão Preto-USP, no período de janeiro a maio de 2001. O grupo de estudo foi constituído de 17 pacientes adultos, independente do sexo, que se encontravam no período transoperatório de cirurgia cardíaca. Os critérios adotados para a inclusão no grupo foram: ser submetido à revascularização do miocárdio, troca ou plastia de valvas e demonstrar interesse na participação do estudo, com assinatura do termo de consentimento pós-informado.

Para a coleta de dados foi elaborado um instrumento, baseado em um modelo conceitual ${ }^{(9)}$ que se fundamenta na Teoria das Necessidades Humanas Básicas $^{(10-11)}$.

A validação de aparência e conteúdo do instrumento foi realizada por seis enfermeiros que atuam na área de cardiologia e trabalham com diagnósticos de enfermagem. Realizada a validação do instrumento iniciamos um pré-teste mediante a aplicação dos instrumentos de coleta de dados em cinco pacientes, que atenderam ao critério de inclusão, com o objetivo de: identificar a melhor forma de aplicação do instrumento, verificar a adequação do conteúdo e identificar problemas que poderiam interferir na fidedignidade dos dados.

Terminado o pré-teste e realizados os ajustes necessários, foi iniciada a coleta de dados, que se procedeu da seguinte maneira: no dia anterior à cirurgia, após consultar a escala cirúrgica e identificar possíveis integrantes do grupo em estudo, os pacientes foram indagados quanto ao interesse em participar do estudo e à necessidade do seu consentimento por escrito.

Considerando a existência de problemas, relacionados ao período pré-operatório, que poderiam interferir diretamente no transoperatório, como, por exemplo, o estado nutricional, o nível de ansiedade entre outros, os pacientes foram avaliados no dia anterior à cirurgia (pré-operatório imediato), seguindo um instrumento de coleta de dados construído e validado para esse fim; no entanto, os problemas identificados nesse período não serão abordados neste momento por não constituírem objetivo deste estudo.

Os pacientes foram avaliados desde a admissão no centro-cirúrgico até o encaminhamento ao centro de recuperação pós-cirurgia cardíaca. Os dados levantados foram submetidos a um processo de raciocínio diagnóstico (análise e síntese) $)^{(12)}$, em seguida, foram estabelecidos os diagnósticos de enfermagem com base na Taxonomia
I da North American Nursing Diagnosis Association $(\text { NANDA) })^{(13)}$.

\section{RESULTADOS}

A Tabela 1 apresenta as categorias diagnósticas identificadas segundo a Taxonomia I da NANDA, no período transoperatório de cirurgia cardíaca.

Tabela 1 - Categorias diagnósticas identificadas em indivíduos adultos no período transoperatório de cirurgia cardíaca. Hospital das Clínicas da Faculdade de Medicina de Ribeirão Preto, janeiro a maio de 2001

\begin{tabular}{lccc}
\hline \multicolumn{1}{c}{ Categoria diagnóstica } & \multicolumn{2}{c}{$\begin{array}{c}\text { Freqüência } \\
\text { (n=17) }\end{array}$} \\
\hline Risco para infecção & 17 & $(100 \%)$ \\
Risco para desequilíbrio no volume de líquidos & 17 & $(100 \%)$ \\
Troca de gases prejudicada & 17 & $(100 \%)$ \\
Risco para aspiração & 17 & $(100 \%)$ \\
Proteção alterada & 17 & $(100 \%)$ \\
Integridade da pele prejudicada & 17 & $(100 \%)$ \\
Risco para disfunção neurovascular periférica & 17 & $(100 \%)$ \\
Risco para lesão perioperatória de posicionamento & 17 & $(100 \%)$ \\
Risco para temperatura corporal alterada & 16 & $(94,1 \%)$ \\
Ansiedade & 07 & $(41,2 \%)$ \\
Hipotermia & 01 & $(5,9 \%)$ \\
\hline
\end{tabular}

Observamos, na Tabela 1, que as categorias diagnósticas risco para infecção, risco para desequilíbrio no volume de líquidos, troca de gases prejudicada, risco para aspiração, proteção alterada, integridade da pele prejudicada, risco para disfunção neurovascular periférica e risco para lesão perioperatória de posicionamento, foram identificadas em todos os pacientes.

Pode-se observar também que apenas um paciente não apresentou risco para temperatura corporal alterada $(94,1 \%)$, por apresentar hipotermia $(5,9 \%)$. O diagnóstico risco para temperatura corporal alterada foi elaborado mediante a apresentação dos fatores de risco indicados na Tabela 4 e da oscilação dos valores da temperatura corporal, porém o paciente em que foi identificada a categoria diagnóstica hipotermia apresentou, além dos fatores comuns aos outros pacientes (como exposição a ambientes frios, inatividade e outros), temperatura corporal abaixo dos níveis considerados normais durante todo o período transoperatório.

A categoria diagnóstica Ansiedade foi identificada em sete pacientes $(41,2 \%)$ que relataram tal sentimento, ainda na recepção do centro cirúrgico. Ressaltamos que esses pacientes foram avaliados antes da administração de medicamentos pré-anestésicos. Isso não ocorreu com o restante do grupo, prejudicando a identificação dessa categoria diagnóstica nos demais 
pacientes.

Estão apresentados na Tabela 2 os fatores relacionados às categorias diagnósticas identificadas no período transoperatório, cuja freqüência foi superior a $50 \%$.

Tabela 2 - Fatores relacionados às categorias diagnósticas reais identificadas em $50 \%$ ou mais dos indivíduos adultos no período transoperatório de cirurgia cardíaca. Hospital das Clínicas da Faculdade de Medicina de Ribeirão Preto, janeiro a maio de 2001

\begin{tabular}{|c|c|c|}
\hline $\begin{array}{l}\text { Categoria } \\
\text { diagnóstica }\end{array}$ & Fatores relacionados & $\begin{array}{c}\text { Freqüência } \\
(n=17)\end{array}$ \\
\hline $\begin{array}{l}\text { Troca de gases } \\
\text { prejudicada }\end{array}$ & $\begin{array}{l}\text { - Desequilíbrio da ventilação- } \\
\text { perfusão }\end{array}$ & 17 (100\%) \\
\hline Proteção alterada & $\begin{array}{l}\text { - Terapias com drogas } \\
\text { (heparinização durante a CEC) }^{*} \\
\text { - Perfis sanguíneos alterados } \\
\text { - Tratamento cirúrgico }\end{array}$ & $\begin{array}{ll}17 & (100 \%) \\
17 & (100 \%) \\
17 & (100 \%)\end{array}$ \\
\hline $\begin{array}{l}\text { Integridade da pele } \\
\text { prejudicada }\end{array}$ & $\begin{array}{l}\text { - Fatores mecânicos (punções } \\
\text { venosas e arteriais e cirurgia) }\end{array}$ & $17(100 \%)$ \\
\hline
\end{tabular}

Nota: As informações assinaladas com asterisco $\left({ }^{*}\right)$ representam as especificidades apresentadas pelos pacientes

Pode-se perceber que, entre os fatores relacionados na Tabela 2 , o desequilíbrio da ventilaçãoperfusão foi identificado em todos os pacientes, sendo o único fator relacionado à troca de gases prejudicada. Isso ocorreu com a integridade da pele prejudicada, relacionada, neste estudo, a fatores mecânicos (punções venosas e arteriais e cirurgia).

Estão apresentadas, na Tabela 3, as características definidoras das categorias diagnósticas de enfermagem identificadas no período transoperatório, cuja freqüência foi superior a $50 \%$.

Tabela 3 - Características definidoras das categorias diagnósticas identificadas em $50 \%$ ou mais dos indivíduos adultos no período transoperatório de cirurgia cardíaca. Hospital das Clínicas da Faculdade de Medicina de Ribeirão Preto, janeiro a maio de 2001

\begin{tabular}{|c|c|c|}
\hline $\begin{array}{c}\text { Categoria } \\
\text { diagnóstica }\end{array}$ & Características definidoras & $\begin{array}{c}\text { Freqüência } \\
(n=17)\end{array}$ \\
\hline $\begin{array}{l}\text { Troca de gases } \\
\text { prejudicada }\end{array}$ & $\begin{array}{l}\text { - Hipercapnia } \\
\text { - Gases arteriais anormais } \\
\text { - pH arterial anormal }\end{array}$ & $\begin{array}{ll}17 & (100 \%) \\
17 & (100 \%) \\
02 & (11,8 \%)\end{array}$ \\
\hline Proteção alterada & $\begin{array}{l}\text { - Alteração neurossensorial (coma } \\
\text { induzido)* } \\
\text { - Coagulação alterada (heparinização } \\
\text { durante a CEC)* } \\
\text { - Imobilidade }\end{array}$ & $\begin{array}{ll}17 & (100 \%) \\
17 & (100 \%) \\
17 & (100 \%)\end{array}$ \\
\hline $\begin{array}{l}\text { Integridade da pele } \\
\text { prejudicada }\end{array}$ & $\begin{array}{l}\text { - Invasão de estruturas no corpo } \\
\text { - Destruição de camadas da pele e } \\
\text { rompimento da superfície da pele }\end{array}$ & $\begin{array}{ll}17 & (100 \%) \\
17 & (100 \%)\end{array}$ \\
\hline
\end{tabular}

Nota: As informações assinaladas com asterisco $\left(^{*}\right)$ representam as especificidades apresentadas pelos pacientes
Observamos na Tabela 3, que todas as características definidoras possuem $100 \%$ de freqüência, com exceção do $p H$ arterial anormal (11,8\%), fator relacionado à categoria diagnóstica troca de gases prejudicada, que foi identificado em apenas dois pacientes.

$\mathrm{Na}$ Tabela 4, apresentamos os fatores de risco das categorias diagnósticas de risco identificadas no período transoperatório, cuja freqüência foi superior a $50 \%$.

Tabela 4 - Fatores de risco dos diagnósticos de enfermagem identificados em $50 \%$ ou mais dos indivíduos adultos no período transoperatório de cirurgia cardíaca. Hospital das Clínicas da Faculdade de Medicina de Ribeirão Preto, janeiro a maio de 2001

\begin{tabular}{|c|c|c|}
\hline Categoria diagnóstica & Fatores de risco & $\begin{array}{c}\begin{array}{c}\text { Freqüência } \\
(\mathrm{n}=17)\end{array} \\
\end{array}$ \\
\hline Risco para infecção & $\begin{array}{l}\text { - Procedimentos invasivos } \\
\text { - Destruição de tecidos } \\
\text { - Exposição ambiental aumentada } \\
\text { - Defesa primária inadequada } \\
\text { - Doença crônica (Diabetes Melitos)* }\end{array}$ & $\begin{array}{ll}17 & (100 \%) \\
17 & (100 \%) \\
17 & (100 \%) \\
17 & (100 \%) \\
07 & (41,2 \%)\end{array}$ \\
\hline $\begin{array}{l}\text { Risco para desequilibrio } \\
\text { no volume de líquidos ** }\end{array}$ & $\begin{array}{l}\text { - (Fatores que interferem nas necessidades de } \\
\text { líquidos)"* } \\
\text { - (Situações em que ocorram perdas de líquidos } \\
\text { corporais ou ganho de líquidos, ou ambos, como } \\
\text { ocorre em cinurgia ou em outros procedimentos } \\
\text { invasivos maiores)" }\end{array}$ & $\begin{array}{l}17(100 \%) \\
17(100 \%)\end{array}$ \\
\hline Risco para aspiração & $\begin{array}{l}\text { - Situações que impedem a elevação da parte } \\
\text { superior do corpo (cirurgia e anestesia)* } \\
\text { - Nível de consciência reduzido (anestesia)* } \\
\text { - Tubo endotraqueal } \\
\text { - Reflexos de náusea e tosse diminuídos }\end{array}$ & $\begin{array}{ll}17 & (100 \%) \\
17 & (100 \%) \\
17 & (100 \%) \\
17 & (100 \%)\end{array}$ \\
\hline $\begin{array}{l}\text { Risco para disfunção } \\
\text { neurovascular periférica }\end{array}$ & $\begin{array}{l}\text { - Trauma (punção artéria radial)* } \\
\text { - Imobilização } \\
\text { - Obstrução vascular }\end{array}$ & $\begin{array}{l}17(100 \%) \\
17(100 \%) \\
12(70,6 \%)\end{array}$ \\
\hline $\begin{array}{l}\text { Risco para lesão } \\
\text { perioperatória de } \\
\text { posicionamento }\end{array}$ & $\begin{array}{l}\text { - Imobilização (durante ato cinúrgico)* } \\
\text { - Distúrbios sensoriais/perceptivos devidos à } \\
\text { anestesia } \\
\text { - Obesidade }\end{array}$ & $\begin{array}{ll}17 & (100 \%) \\
17 & (100 \%) \\
07 & (41,2 \%)\end{array}$ \\
\hline $\begin{array}{l}\text { Risco para temperatura } \\
\text { corporal alterada }\end{array}$ & $\begin{array}{l}\text { - Trauma afetando a regulação da temperatura } \\
\text { (cirurgia e CEC) }^{\star} \\
\text { - Vestimentas inadequadas para temperatura } \\
\text { ambiental } \\
\text { - Inatividade } \\
\text { - Sedação } \\
\text { - Exposição a ambiente frio (sala cinúrgica) } \\
\text { - Extremos de peso (obesidade)* } \\
\text { - Medicações que causam vasodilatação }\end{array}$ & $\begin{array}{l}16(94,1 \%) \\
16(94,1 \%) \\
16(94,1 \%) \\
16(94,1 \%) \\
16(94,1 \%) \\
06(35,3 \%) \\
04(23,5 \%)\end{array}$ \\
\hline
\end{tabular}

Nota: As informações assinaladas com asterisco $\left({ }^{*}\right)$ representam as especificidades apresentadas pelos pacientes. As informações assinaladas com dois asteriscos $\left({ }^{* *}\right)$ representam os fatores de risco estabelecidos pelas pesquisadoras

Para as categorias risco para infecção e risco para aspiração, foram identificados, para cada uma, quatro fatores relacionados com freqüência igual a 100\%.

A categoria diagnóstica de risco para temperatura corporal alterada apresentou, como principais fatores de risco, trauma afetando a regulação da temperatura (cirurgia e CEC), vestimentas inadequadas para temperatura ambiental, inatividade, sedação e exposição a ambiente 
frio (sala cirúrgica), identificados em $94,1 \%$ dos pacientes.

Para o estabelecimento da categoria diagnóstica risco para desequilíbrio no volume de líquidos, foram agrupados dados relativos ao volume hídrico infundido durante a cirurgia, ao volume de diurese, as perdas sensíveis e insensíveis e aos procedimentos inerentes à cirurgia como, por exemplo, a CEC. Esses dados, associados à definição do diagnóstico, ajudaram-nos a diferenciá-los de outros diagnósticos similares, pois os fatores de risco para esse diagnóstico ainda não foram determinados pela NANDA. Portanto, apontamos para essa categoria os fatores de risco: fatores que interferem nas necessidades de líquidos e situações em que ocorram perdas de líquidos corporais ou ganho de líquidos, ou ambos (como ocorre em cirurgia ou em outros procedimentos invasivos maiores); identificados em todos os pacientes avaliados.

O risco para lesão perioperatória de posicionamento apresentou como fatores de risco a imobilização (durante ato cirúrgico) e os distúrbios sensoriais/perceptivos devidos à anestesia, identificados em todos os pacientes. A obesidade também é fator de risco para esse diagnóstico, no entanto foi identificada com menor freqüência $(41,2 \%)$.

\section{DISCUSSÃO}

Observamos que, das 11 categorias diagnósticas identificadas (Tabela 1$)$, oito $(72,7 \%)$ foram identificadas em todos os pacientes. Essa elevada incidência de pacientes com os mesmos diagnósticos talvez seja resultante do intenso controle que o anestesista, perfusionista (profissional responsável pela CEC) e cirurgião têm dos mecanismos fisiológicos do paciente no período intra-operatório. O paciente, nesse período, é submetido a uma série de "situações artificiais" como: indução do coma (agentes anestésicos); indução de parada cardiorrespiratória (cardioplegia); inibição do sistema da coagulação (heparinização sistêmica); oxigenação e fluxo sangüíneos artificiais (CEC) e indução da hipotermia, podendo acarretar problemas similares a todos os pacientes.

Uma outra questão que merece destaque é o fato de $90,9 \%$ dos diagnósticos, identificados no transoperatório estarem relacionados às necessidades fisiológicas; isso poderia ser esperado, uma vez que o indivíduo que se encontra em um processo de doença, tem, como principal necessidade, a sobrevivência. As necessidades fisiológicas ou de sobrevivência são as de maior prioridade, pois são as que, em geral, os indivíduos procuram satisfazer em primeiro lugar ${ }^{(10)}$. Na literatura, também observamos uma predominância dos diagnósticos de enfermagem relacionados às necessidades psicobiológicas $^{(8)}$.

A seguir, apresentamos a discussão dos diagnósticos de enfermagem encontrados com freqüência superior a $50 \%$.

Risco para infecção

A cirurgia, além de romper a barreira epitelial, interrompendo a chegada ao tecido de glicose, aminoácido e oxigênio, desencadeia uma série de reações sistêmicas que facilitam a ocorrência de um processo infeccioso. No sítio operatório, ocorre hipóxia, alteração do pH e deposição de fibrina. A hipóxia e a acidose dificultam a migração dos neutrófilos e sua atividade microbicida. Por sua vez, a deposição de fibrina contribui com a patogênese da infecção, devido à sua habilidade em seqüestrar bactérias e afetar os mecanismos locais de defesa ${ }^{(14)}$.

Além dos procedimentos invasivos e da defesa primária insuficiente provocada pelo trauma cirúrgico, vários outros fatores influenciam na incidência de infecção na ferida operatória, entre eles as condições clínicas préoperatórias do paciente (idade, estado nutricional, doenças crônicas, etc.), as condições técnicas em que a cirurgia foi realizada e a permanência hospitalar pré-operatória (quanto mais longa, maior a chance de infecção cirúrgica, pela substituição da flora do paciente pela flora hospitalar $)^{(14)}$. Não podemos nos esquecer, também, dos fatores relacionados à CEC (seqüestro e destruição de leucócitos).

Risco para desequilíbrio no volume de líquidos

As alterações hidroeletrolíticas podem ocorrer devido ao sangramento, às perdas insensíveis (que podem chegar a $8 \mathrm{ml} / \mathrm{kg} / \mathrm{h}$ ), a distúrbios renais e a hemodiluição utilizada na CEC. A hemodiluição empregada na CEC consiste na administração de 1200 a 2400 ml de cristalóide, com o objetivo de reduzir a viscosidade sangüínea 
(permitindo ao sangue fluir com mais facilidade) e reduzir a resistência vascular periférica, resultando em um aumento da perfusão tissular ${ }^{(15-17)}$.

Nesse período, podem ocorrer a sobrecarga hídrica, a hipovolemia e os distúrbios eletrolíticos. A sobrecarga hídrica decorre da infusão de grande quantidade de líquidos, associada à CEC e à hemodiluição. Essa sobrecarga hídrica é intensificada pelos níveis de hormônio antidiurético, normalmente elevados no transoperatório devido à CEC, à anestesia e ao estresse cirúrgico ${ }^{(17-18)}$. A hipovolemia caracteriza-se por uma diminuição do volume intravascular e pode ser resultado de uma reposição inadequada de volume ou do seqüestro de líquido para o espaço intersticial ${ }^{(18)}$. As alterações eletrolíticas podem ocorrer em decorrência da hemodiluição utilizada na CEC, sendo as alterações mais importantes, as ocorridas com o potássio, cálcio e magnésio, responsáveis entre outras, pela transmissão e condução de impulsos nervosos e pela contração do músculo cardíaco ${ }^{(18)}$.

Troca de gases prejudicada

Os principais distúrbios no trans e pós-operatório de cirurgia cardíaca são resultantes da CEC; esse é o fator que diferencia os pacientes de cirurgia cardíaca dos pacientes submetidos a outras cirurgias de grande porte. Durante a CEC, as trocas gasosas ocorrem mediante contato direto do sangue com o gás existente nos oxigenadores artificiais, podendo acarretar excesso ou déficit de oxigênio e/ou gás carbônico.

\section{Risco para aspiração}

A dificuldade de elevar a parte superior do corpo, a depressão dos reflexos da tosse e deglutição e o aumento da pressão intragástrica, causados pelos agentes anestésicos, podem levar à aspiração de secreções. As conseqüências dessa situação podem ser desastrosas para o paciente, pois a aspiração do conteúdo gástrico pode provocar irritação e destruição da mucosa traqueal e pneumonias, aumentando o risco de infecção.

\section{Proteção alterada}

O paciente submetido à cirurgia cardíaca apresenta proteção alterada devido aos procedimentos inerentes à cirurgia e especialmente devido à CEC. Ao contrário da maioria dos procedimentos invasivos, a CEC produz significativas alterações no equilíbrio fisiológico do organismo, constituindo-se num agente agressor complexo e multifatorial ${ }^{(17)}$.

A proteção alterada está relacionada a terapias com drogas (heparinização durante a CEC), a perfis sangüíneos alterados e ao tratamento cirúrgico (Tabela 2).

Dentre as alterações produzidas pela CEC estão a inibição do sistema de coagulação (heparinização sistêmica) e as alterações nas células sangüíneas, decorrentes do contato do sangue com superfícies estranhas, isto é, não endoteliais, como os oxigenadores e circuitos ${ }^{(17)}$. A heparinização sistêmica é realizada na CEC, com a finalidade de inibir as fases finais da cascata de coagulação sangüínea, permitindo o contato do sangue com as superfícies dos oxigenadores e circuitos sem a formação de coágulos ${ }^{(17)}$.

Durante a CEC, as células sangüíneas estão sujeitas à ação de diversas forças que diferem das forças que atuam na circulação normal. As hemácias, os leucócitos e as plaquetas são afetados pela CEC, tanto pelo trauma físico, quanto pelo contato com as superfícies do circuito. Os leucócitos são, em parte, seqüestrados da circulação, muitos leucócitos aderem aos tubos do circuito e outros são destruídos, diminuindo, com isso, a defesa do organismo contra infecções. Além de seqüestrar os leucócitos da circulação, a CEC inibe significativamente a sua propriedade de fagocitar os germes invasores do organismo $^{(17)}$.

Integridade da pele prejudicada e risco para lesão perioperatória de posicionamento

A categoria diagnóstica integridade da pele prejudicada é definida como estado no qual um indivíduo tem a epiderme e/ou derme alteradas, foi uma categoria comum a todos os pacientes, relacionada a fatores mecânicos, secundários aos procedimentos cirúrgicos ${ }^{(13)}$.

O paciente submetido à cirurgia cardíaca permanece imóvel por um longo período, considerando o prolongado tempo intra-operatório (aproximadamente cinco horas), aumentando, com isso, a susceptibilidade em adquirir uma lesão perioperatória de posicionamento (úlcera de pressão). Além disso, os agentes anestésicos interferem na vasodilatação e constrição normal, reduzindo, 
assim, a perfusão para as proeminências ósseas e para regiões que se encontram sob pressão(18). Devemos considerar, ainda, os fatores relacionados à CEC, que levam à vasoconstrição periférica e à diminuição da oxigenação tecidual devido à hipotermia induzida.

Risco para disfunção neurovascular periférica

O risco para esse diagnóstico está relacionado ao fato de que muitos pacientes com doença arterial coronariana são portadores de aterosclerose e podem apresentar obstrução vascular em outros locais. Um outro fator é o prolongado tempo em que o paciente permanece imóvel podendo levar a uma trombose venosa profunda (TVP), principalmente em pacientes que possuem trajetos varicosos. Ainda, um outro fator a ser considerado é a cateterização arterial feita preferencialmente nas artérias radial e femoral, para medida direta da pressão arterial, podendo acarretar complicações, entre elas, a obstrução arterial (trombose), embolia e isquemia ${ }^{(19)}$.

Risco para temperatura corporal alterada

O risco para temperatura corporal alterada é definido como "estado no qual um indivíduo corre o risco de não conseguir manter a temperatura corporal dentro dos parâmetros normais"”(13).

No período transoperatório de cirurgia cardíaca o paciente apresenta oscilações de temperatura devido às alterações no equilíbrio fisiológico causado pela CEC. No início da cirurgia, o paciente encontra-se geralmente hipotérmico, devido ao prolongado tempo exposto à baixa temperatura da sala cirúrgica (abertura de cavidades serosas), aos agentes anestésicos, o que impede o organismo de reagir à redução de temperatura corpórea por meio da contração muscular, capaz de produzir calor, à infusão de líquidos a baixa temperatura, à ação dos potentes agentes vasodilatadores (nitroprussiato de sódio) utilizados para controle da pressão arterial e devido à hipotermia induzida na CEC. A hipotermia tem sido amplamente utilizada durante a CEC com o objetivo de reduzir o consumo de oxigênio pelos tecidos e aumentar a viabilidade tecidual se o suprimento de sangue for interrompido ou diminuído, protegendo, com isso, os órgãos vitais ${ }^{(16)}$. Ao final da CEC, o paciente é aquecido gradualmente, mediante utilização de um colchão térmico, com o objetivo de fazer com que o paciente retorne a sua temperatura normal.

\section{CONCLUSÕES}

Os resultados encontrados neste estudo permitiram-nos concluir:

- das 11 categorias diagnósticas identificadas, seis $(54,5 \%)$ correspondem a categorias de risco, e cinco, $(45,5 \%)$ a categorias diagnósticas reais;

- das categorias diagnósticas reais, três apresentaram freqüência de $100 \%$, sendo as demais identificadas em menos de $50 \%$ dos sujeitos;

- das 11 categorias diagnósticas identificadas, oito (72,7\%) foram identificadas em todos os pacientes;

- 90,9\% das categorias diagnósticas estão relacionados às necessidades fisiológicas.

\section{CONSIDERAÇÕES FINAIS}

Devemos ressaltar a importância da avaliação préoperatória na identificação dos diagnósticos de enfermagem, uma vez que o paciente poderá apresentar problemas no pré-operatório, que poderão interferir diretamente na identificação dos diagnósticos de enfermagem no período transoperatório. Por exemplo: um paciente, no período pré-operatório, refere perda da sensibilidade em membro superior direito; baseando-se nessa evidência, poderá ser estabelecido, nos períodos posteriores (trans e pós-operatório), o diagnóstico de enfermagem risco para lesão. Tal diagnóstico poderia não ser identificado se o paciente fosse avaliado somente no período transoperatório, pois essa informação (perda da sensibilidade em membro superior direito) poderia ser omitida, considerando-se o fato de o paciente estar sonolento devido aos medicamentos pré-anestésicos.

Acreditamos que o presente estudo contribuirá para o planejamento do cuidado a pacientes que se encontram no período transoperatório, resultando na implementação de ações rápidas e eficazes para a resolução dos problemas identificados. "Diagnósticos de enfermagem comuns a um grupo de indivíduos delineiam o perfil deste grupo, permitindo um direcionamento global das intervenções de enfermagem"(20). 


\section{REFERÊNCIAS BIBLIOGRÁFICAS}

1. Lotufo PA. Epidemiologia das doenças cardíacas no Brasil: histórico, situação atual e proposta de modelo teórico. Rev Soc Cardiol Estado de São Paulo 1996; 6:541-7.

2. Conselho Regional de Enfermagem do Estado de São Paulo (Coren-SP). Documentos básicos de enfermagem principais leis e resoluções que regulamentam o exercício profissional de enfermeiros, técnicos e auxiliares de enfermagem. São Paulo; 2000.

3. Rossi LA. O processo de enfermagem em uma unidade de queimados: análise e reformulação fundamentado na metodologia da problematização. [Dissertação]. Ribeirão Preto (SP): Escola de Enfermagem de Ribeirão Preto/USP; 1992.

4. Jesus CAC. Raciocíno clínico de graduandos e enfermeiros na construção de diagnósticos de enfermagem. [Tese]. Ribeirão Preto (SP): Escola de Enfermagem de Ribeirão Preto/ USP; 2000.

5. Leite JL. Necessidades e expectativas do paciente em pós-operatório de cirurgia cardíaca. Rev Bras Enfermagem $1985 \mathrm{jul} / \mathrm{dez} ; 38(3 / 4): 238-44$.

6. Pagliuca LMF. Necessidades humanas básicas afetadas em um grupo de pacientes em pós-operatório de cirurgia cardíaca na UTI. Rev Esc Enfermagem USP 1988 agosto; 22(Número especial):83-102.

7. Faria MFG. Diagnósticos de enfermagem respiratórios em pacientes cardíacos cirúrgicos. [Dissertação].Ribeirão Preto (SP): Escola de Enfermagem de Ribeirão Preto/USP; 2000. 8. Killen AR, Kleinbeck SVM, Golar K, Takahashi Schuchardt $J$, Uebele J. The prevalence of perioperative nurse clinical judgments. AORN J 1997; 65(1):101-8.

9. Horta WA. A observação sistematizada na identificação dos problemas de enfermagem em seus aspectos físicos. Rev Bras Enfermagem 1974; 27(2):214-9.

10. Maslow AH. Motivation and personality. $2^{\mathrm{a}}$ ed. New York: Harper \& Row; 1970.

11. Mohana J. O mundo e eu. $2^{\mathrm{a}}$ ed. Rio de Janeiro: Agir; 1964. 12. Risner PB. Diagnosis: analysis and synthesis of data. In: Christensen PJ, Kenney JW. Nursing process: conceptual models. $4^{a}$ ed. St. Louis: Mosby; 1990. p.132-57.

13. NANDA - North American Nursing diagnosis Association. Diagnósticos de enfermagem: definições e classificações. Porto Alegre: Artes Médicas; 2000.

14. Rabhae GN, Ribeiro N Filho, Fernandes AT. Infecção do sítio cirúrgico. In: Fernandes AT, Fernandes MOVF. Infecção hospitalar e suas interfaces na área de saúde. São Paulo: Atheneu; 2000. p.479-502.

15. Senra DF, lasbech JÁ, Oliveira SA. Pós-operatório em cirurgia cardíaca de adultos. Rev Soc Cardiol Estado de São Paulo 1998 maio/jun.; 8(3):446-54.

16. Teixeira GF Filho, Batista G. Fisiopatologia da circulação extracorpórea. In: Teixeira GF Filho. Temas atuais em circulação extracorpórea. Porto Alegre: Sociedade Brasileira de Circulação Extracorpórea; 1997. p. 22-8.

17. Souza MHL, Elias DO. Resposta inflamatória sistêmica à circulação extracorpórea In: Souza MHL, Elias DO. Fundamentos da circulação extracorpórea. Porto Alegre: Artes Médicas; 1999. p.374-91.

18. Fortuna ABP. Pós-operatório imediato em cirurgia cardíaca. 2a ed. São Paulo: Atheneu; 1992.

19. Alvarenga GS, Kopel L, Carvalho RT, Lage SG. Suporte inotrópico e monitorização hemodinâmica na insuficiência cardíaca grave. Rev Soc Cardiol Estado de São Paulo 1998 maio/jun.; 8(3):455-63.

20. Cruz DALM. Os diagnósticos de enfermagem no ensino e na pesquisa. Rev Esc Enfermagem USP 1992 dezembro; 26(3):427-34. 